\title{
Simple intradyne PSK system for udWDM-PON
}

\author{
Josep Prat, ${ }^{1 *}$ Victor Polo, ${ }^{1}$ Panagiotis Zakynthinos, ${ }^{2}$ Ivan Cano,,${ }^{1}$ Jeison Tabares, ${ }^{1}$ \\ Josep M. Fàbrega, ${ }^{3}$ Dimitrios Klonidis, ${ }^{2}$ and Ioannis Tomkos ${ }^{2}$ \\ ${ }^{1}$ Department of Signal Theory and Communications, Universitat Politècnica de Catalunya; Jordi Girona D4; 08034 \\ Barcelona, Spain \\ ${ }^{2}$ Athens Information Technology Center, 19.5km Markopoulo Ave., Peania, 19902, Athens, Greece \\ ${ }^{3}$ Centre Tec. de Telecom. de Catalunya (CTTC), 08860, Castelldefels, Spain \\ iprat@tsc.upc.edu
}

\begin{abstract}
A homodyne coherent receiver for ultra-dense WDM-PON with off the shelf components is presented. It consists of a conventional DFB, phase switched clock signal, an optical coupler instead of a $90^{\circ}$ hybrid, balanced photodetectors and digital signal processing. The phase swing for a DBPSK signal was optimized and the performance was experimentally evaluated in terms of the sensitivity for several laser linewidths. The acceptable frequency offset and clock time delay was also assessed. The results exhibit a sensitivity of $-48 \mathrm{dBm}$ at a BER of $10^{-3}$ and indicate a high tolerance to phase noise.
\end{abstract}

(C)2012 Optical Society of America

OCIS codes: (060.0060) Fiber optics and optical communications; (060.1660) Coherent communications.

\section{References and links}

1. K. Y. Cho, U. H. Hong, and A. Agata, "10-Gb/s, 80-km reach RSOA-based WDM PON employing QPSK signal and self-homodyne receiver,” Proc. OFC'12, paper OW1B.1, (2012)

2. R. Rodes, N. Cheng, J. B. Jensen, and I. Tafur, " $10 \mathrm{~Gb} / \mathrm{s}$ real-time all-VCSEL low complexity coherent scheme for PONs,” Proc. OFC’12, paper OTh4G.2 (2012)

3. D. Lavery, R. Maher, D. Millar, B. C. Thomsen, P. Bayvel, and S. Savory, "Demonstration of 10 Gbit/s colorless coherent PON incorporating tunable DS-DBR lasers and low-complexity parallel DSP,” Proc. OFC’12, paper PDP5B.10 (2012)

4. H. Rohde, S. Smolorz, S. Wey, and E. Gottwald, “Coherent optical access networks,” Proc. OFC'11, paper OTuB1 (2011)

5. B. Schrenk, J. M. Fabrega, C. Kazmierski, J. Lázaro, and J. Prat, "SOA/REAM as vector modulator for QAM upstream,” Proc. OFC'11, paper OThK1 (2011)

6. L. G. Kazovsky, G. Kalogerakis, and W. Tao, "Homodyne phase-shift-keying systems: past challenges and future opportunities,” J. Lightwave Technol. 12, 4876-4884, (2006)

7. J. Prat, and J. M. Fabrega, "New homodyne receiver with electronic I\&Q differential demodulation,” Proc. ECOC'05, paper We4.P104, (2005)

8. J. M. Fabrega, and J. Prat, "Experimental investigation of channel crosstalk in a time-switched phasediversity optical homodyne receiver,” Opt. Lett. 34, 452-454 (2009)

9. J. M Fabrega and J. Prat, "Homodyne receiver prototype with time-switching phase diversity and feedforward analog processing,” Opt. Lett. 32, 463-465 (2007)

10. I. Cano, M. C. Santos, V. Polo, and J. Prat, "Dimensioning of OFDMA PON with non-preselectedindependent ONUs sources and wavelength control,” proc. ECOC'11, Tu.E.C.2. (2011)

11. D. Piehler, "PICs in PONs," proc. OFC'12, paper NTu1J.6. (2012)

12. A. Ramaswamy, L. A. Johansson, J. Klamkin, C. Sheldon, H. F. Chou, M. J. Rodwell, L. A. Coldren, and J. E. Bowers, “Coherent receiver based on a broadband optical phase-lock loop," Proc. OFC’07, paper PDP3, (2007)

\section{Introduction}

Homodyne/intradyne coherent reception has resurrected as a feasible approach for ultradense-WDM transport and access networks. In Fiber-to-the-Home (FTTH) passive optical networks (PONs) it enables compatibility with current splitter-based fiber infrastructure and is 
a promising candidate to provide high optical bandwidth [1-5]. Heterodyne optical receivers can be a first approach to coherent detection but it presents inherent image frequency interference. As a result, homodyne or intradyne (without optical phase lock) reception is considered a better solution [6-8].

A new PSK receiver architecture based on time-switching phase-diversity was experimentally demonstrated in [7]. It was a first approach towards a low-cost implementation of a reliable optical homodyne receiver. It showed a $1.8 \%$ linewidth/bit-rate ratio tolerance operating in real time, the maximum then reported. For that system, the channel spacing was 3 $\mathrm{GHz}[7,8]$ and all the processing in the receiver was performed with analog hardware requiring tuning and critical adjustments.

In this paper, we present an enhancement of the intradyne system by means of digital signal processing (DSP), direct phase scrambling, and a further simplification of the architecture. The DSP performs demodulation, decoding and filtering with improved quality and flexibility; and thus can be a potential low-cost solution to offer bidirectional $1 \mathrm{~Gb}$ Ethernet to each of hundreds of homes in a PON. Two architectures for processing are tested giving identical results. In addition, the phase swing is controlled and optimized to get the Inphase (I) and quadrature (Q) component energy at the first and second half of the bit respectively. This greatly simplifies the phase-diversity receiver hardware and enables high phase noise tolerance. The receiver is evaluated in terms of its sensitivity and tolerance to phase noise. The results indicate an improvement of around $4 \mathrm{~dB}$ in the sensitivity when compared to previous experiments [7,9] in which the receiver employed analog signal processing.

\section{Network architecture}

The network architecture (Fig. 1) is based on a standard PON splitter-based tree. Channel selection is not made in the time-domain (TDM) but in the optical frequency domain (WDM) with ultra-dense $\mathrm{GHz}$ channel spacing (udWDM) and electrical channel filtering. Following $[10,11]$, the wavelength grid is not kept standard but left flexible for best adaptation to each ONU laser center wavelength to offer the maximum spectral efficiency. A high-resolution spectral analyzer at the OLT monitors and controls the down- and up- stream wavelengths. In this work, we focus on the up-stream direction, as is the most challenging in PONs (the downstream can use another waveband or a remodulation strategy tolerant to Rayleigh-scattering and reflections [1].

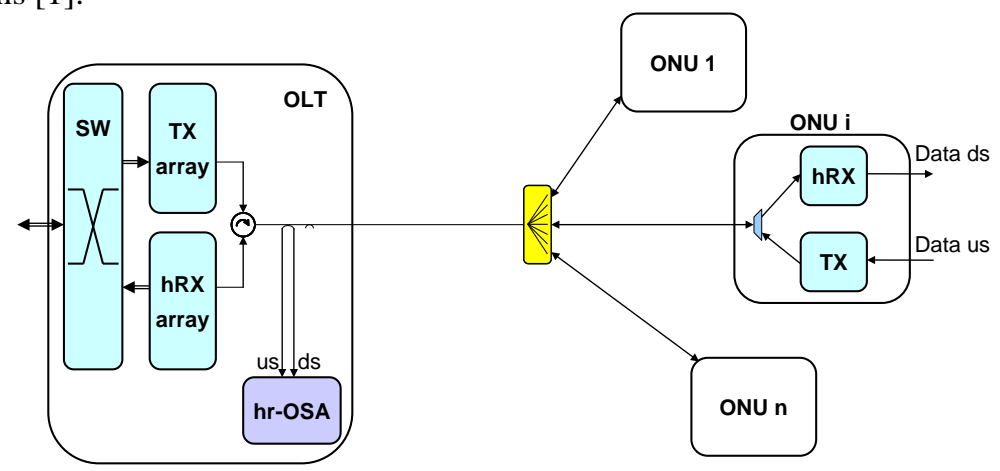

Fig. 1. Generic PON architecture.

\section{Receiver scheme}

The proposed diversity receiver, shown in Fig. 2(a), has two main parts: the first consists of a coherent photo-receiver with an added clock-synchronous optical phase swing $\left(0-\sim 90^{\circ}\right)$. The second part is digital post-processing which performs the signal demodulation and synchronous combination of the orthogonal I\&Q components. The local laser does not need to 
be phase-coherent with the incoming optical carrier, although an automatic wavelength controller is required to maintain the two wavelengths close to each other. Also, a polarization controller is used to compensate signal fluctuations due to state of polarization (SOP) changes, although in a practical deployment a polarization diversity scheme or a fast polarization controller has to be included at the OLT [6].

The coherent photo-receiver mixes the incoming signal optical field with the scrambled local laser carrier in the balanced photo-detector stage. The local laser is externally modulated with a phase modulator controlled by the filtered data clock, producing a phase change to beat the I and Q signal energy at the first and second half of each bit time $\left(T_{b}\right)$ respectively.

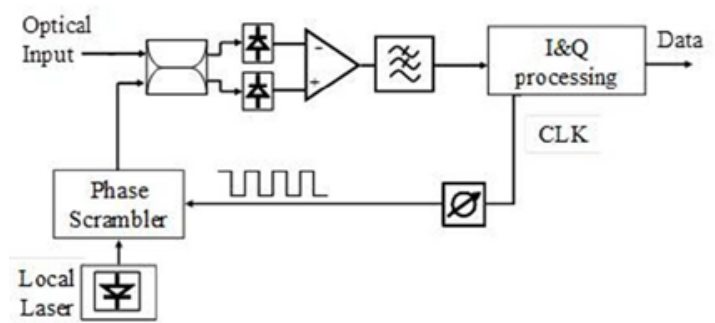

(a)

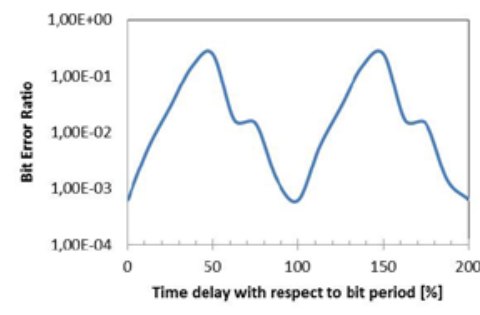

(b)

Fig. 2. (a) Receiver module schematics, (b) BER against clock delay with respect to $T_{b}$

After the conventional balanced photodetector an electrical filter is placed to reduce the noise and reject the interference from adjacent WDM channels. Due to the synchronous $0-90^{\circ}$ phase scrambling, at this point the signal has twice the bandwidth of the low-pass equivalent data power spectrum. It is important to note that the extracted data clock is relevant in this operation, as synchronizing the optical phase swing. This is noted in Fig. 2(b) where a small delay in the clock signal with respect to the bit reduces the bit error ratio (BER).

After filtering, the samples are sent to a DSP module. This is the core of the receiver and it can be more or less complex depending on the modulation format used. Two processing techniques were studied for detecting differentially coded PSK. The first one, illustrated in Fig. 3(a), consisted of a delay and multiply block for comparing the phase variation with respect to the previous bit, and subsequently combined both $I$ and $Q$ components after a $T_{b} / 2$ delay. The second method, Fig. 3(b), involves a switch interchanging every $T_{b} / 2$ to separate the I and Q sections of the received signal in order to lower the electrical bandwidth needed for each stream. Both components are delayed and multiplied in its corresponding branch. Then, the I part is further delayed by $\mathrm{T}_{\mathrm{b}} / 2$ and combined with the $\mathrm{Q}$ part. The advantage of these simple techniques is that they reduce the complexity of the phase noise impairment to likely a phase cycle-slipping in the bounds of a bit time, possible when using high linewidth lasers. The subdivision of the bit period doubles the bandwidth, posing a theoretical penalty of $3 \mathrm{~dB}$ compared with standard $90^{\circ}$ hybrid and 4 photodiodes, but their simpler implementation makes them attractive for access networks.

The two techniques were tested with data obtained experimentally. White noise was added to the samples to compare their performance in terms of the BER for several signal to noise ratios (SNR). The results are plotted in Fig. 3(c). Both techniques perform identical, however method 1 requires less components and is simpler to implement. Hence we employed it for the rest of the experiments. Furthermore, a similar opto-electronic function has been integrated in an $\mathrm{InP}$ substrate revealing its potential low-cost production [12]. 


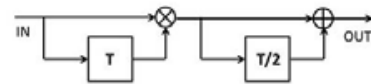

(a)

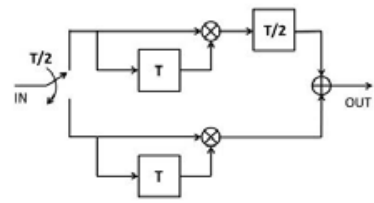

(b)

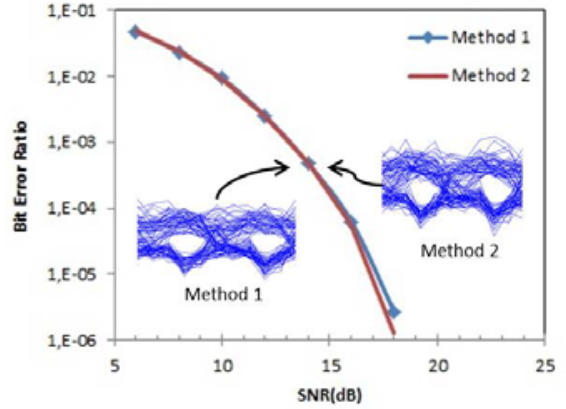

(c)

Fig. 3. I\&Q processing (a) method 1and (b) method 2 schematics, (c) BER against SNR evaluation for both methods.

\section{Experimental setup}

For the experimental setup, the transmitted data consisted of a non return to zero (NRZ) $2^{7}-1$ pseudo random binary sequence (PRBS). It was grouped in 2000 blocks of 512 bits and stored in an arbitrary waveform generator (AWG) operating at $1.25 \mathrm{Gbps}$. The data was adequately amplified and modulated by a MZM biased at the null-point to get a binary $0-180^{\circ}$ PSK waveform. The optical signal was then launched through $25 \mathrm{~km}$ of single-mode fiber. For the proof of concept, and to avoid the need of an automatic frequency control, the same Tx laser was used as local oscillator (LO), set in the receiver at a power of $-10 \mathrm{dBm}$. Since the fiber decorrelated the transmitted optical signal and the LO, realistic phase noise was present in the Rx signal. Next to the $\mathrm{LO}$, a $\mathrm{LiNbO}_{3}$ phase modulator was placed and properly driven by a clock signal stored in the AWG, for achieving the desired optical phase swing. The homodyne receiver implemented is shown in Fig. 2(a). The electrical signal at the output of the photodiodes was sampled with a $50 \mathrm{GSa} / \mathrm{s}$ real-time oscilloscope at $12.5 \mathrm{GSa} / \mathrm{s}$ with 10 samples per bit. Therefore the signal consisted of 5 samples for each I and Q component. Only the even samples of each component were considered afterwards, giving a total of 4 samples per bit which were then post-processed with the I\&Q method 1 described above in Matlab ${ }^{\circledR}$. Due to the amount of memory available, the minimum measurable BER was $10^{-6}$, well below the FEC threshold. The complete experimental setup is depicted in Fig. 4.

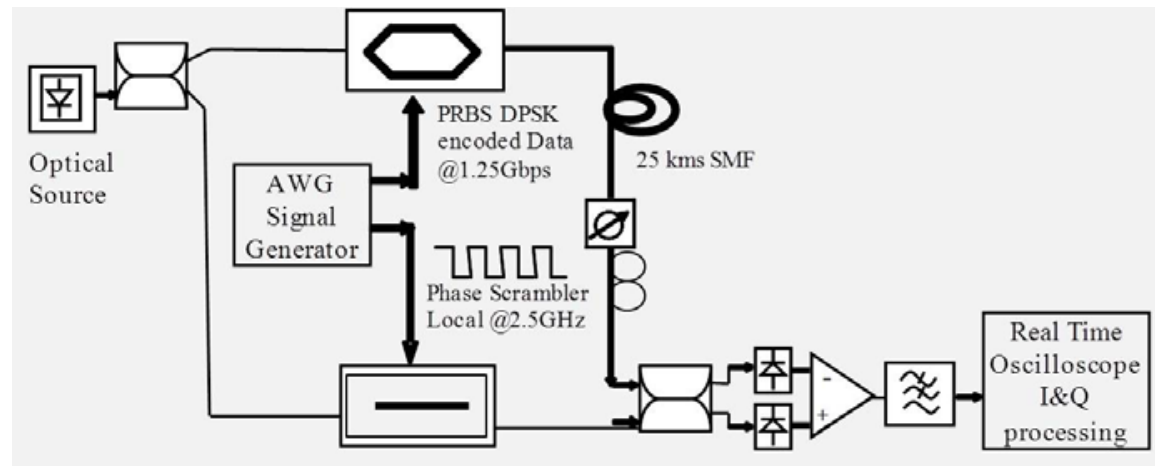

Fig. 4. Experimental set-up

\section{System performance}

The system performance was tested measuring the BER by counting the errors in the received bits, as a function of the input power to the receiver. As the overall operation is affected by the specific waveform $[7,9]$ several phase swings values were considered for optimization. The calibration was performed using a narrow linewidth $(100 \mathrm{kHz})$ external-cavity-laser (ECL). The results are shown in Fig. 5(a) for several received optical powers. Likewise, in 
Fig. 5(b) are plotted the results when using ideal components in a simulation environment. The difference in the sensitivity values between the experimental and the simulated curves are because of the ideal components considered in the second case.

The optimum amplitude was of $4.2 \mathrm{Vpp}(\mathrm{V} \pi=11.5 \mathrm{~V})$ which corresponds to a phase swing of about $66^{\circ}$ degrees. This relation was calibrated using the fundamental tone cancellation method. The optimum phase swing for a wide input power range is lower than the theoretical $90^{\circ}$ for a squared signal as observed in Fig. 5(a). The phase swing is also lower than previous values with simplified analog processing and a sinusoidal clock signal reported in [7 - 9]. This difference is because of an offset caused by a filter in the clock signal. When considering this effect, the optimum values shift close to $70^{\circ}$ as noticed in the dotted curves in Fig. 5(b).

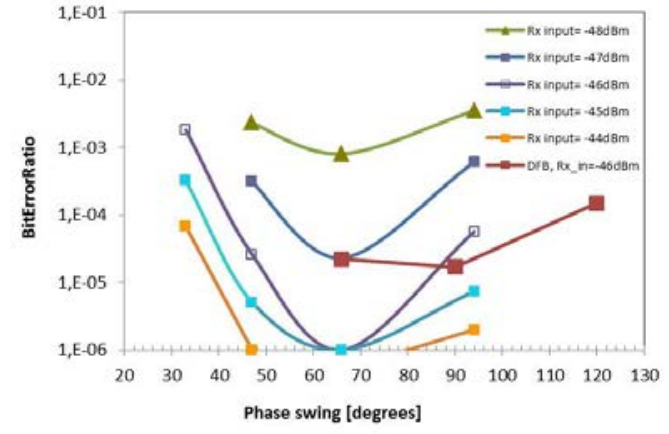

(a)

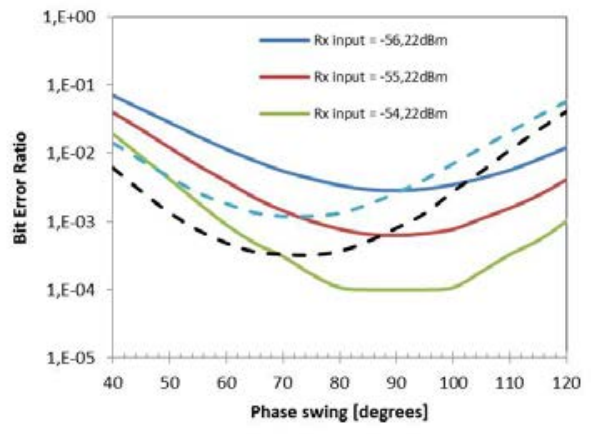

(b)

Fig. 5. BER against phase swing for several received optical powers, (a) experimental results, (b) simulation results with ideal (solid) and offset (dotted) clock signal with $100 \mathrm{kHz}$ laser linewidth

The experimental phase swing for a DFB laser is also presented in Fig. 5(a). In this case, the optimum appears at about $90^{\circ}$ phase swing. However, the curve is very flat, showing a small penalty when compared with the 66 degrees. This is because of the BER floor obtained for such a laser.

In order to determine the sensitivity of the system and to confirm the optimum phase swing, BER data was plotted varying the Rx input power for several phase swings using the $100 \mathrm{kHz}$ linewidth ECL. Figure 6 shows these results, giving a sensitivity of $-48 \mathrm{dBm}$ at a BER of $10^{-3}$ and $-46 \mathrm{dBm}$ at BER of $10^{-6}$. These results improve the ones reported in [9] by about $4 \mathrm{~dB}$.

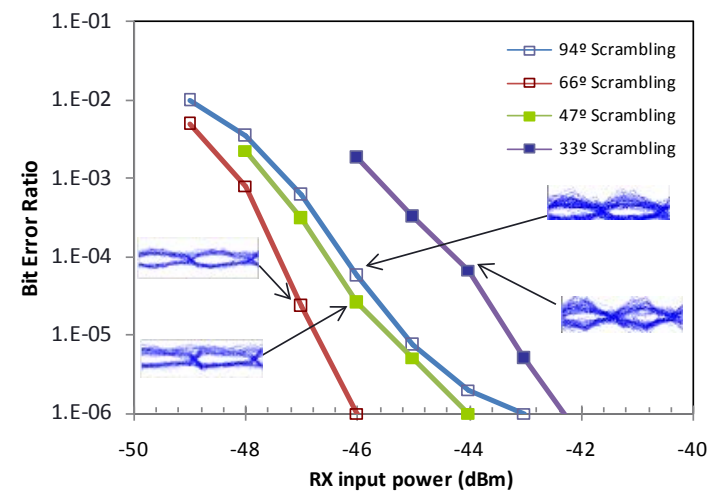

Fig. 6. Receiver sensitivity for several phase swings.

\#177342 - \$15.00 USD

(C) 2012 OSA 
The laser linewidth tolerance of the system was also evaluated. Different lasers were used as light sources with datasheet specified linewidths going from $100 \mathrm{kHz}$ (ECL) up to $15 \mathrm{MHz}$ (DFB). The BER measures for several lasers are plotted in Fig. 7(a). The legends consider the total linewidth with the Tx and Rx lasers together. For a BER of $10^{-3}$ there is a penalty of almost $1 \mathrm{~dB}$ when increasing the total linewidth from $200 \mathrm{kHz}$ to $2 \mathrm{MHz}$, and further enlarges to $2 \mathrm{~dB}$ and almost $5 \mathrm{~dB}$ for a $4 \mathrm{MHz}$ and $30 \mathrm{MHz}$ laser respectively. For a BER of $10^{-6}$, the penalties are of $2.8 \mathrm{~dB}$ and $4 \mathrm{~dB}$ for $2 \mathrm{MHz}$ and $4 \mathrm{MHz}$ linewidths compared with the one of $100 \mathrm{kHz}$.

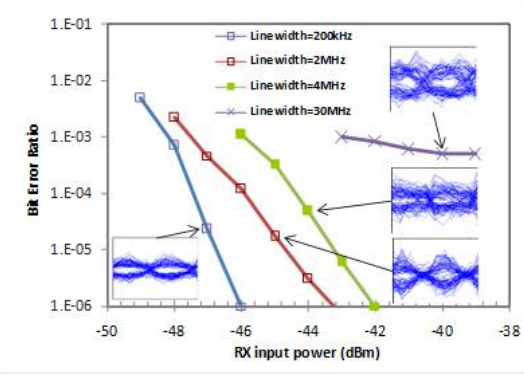

(a)

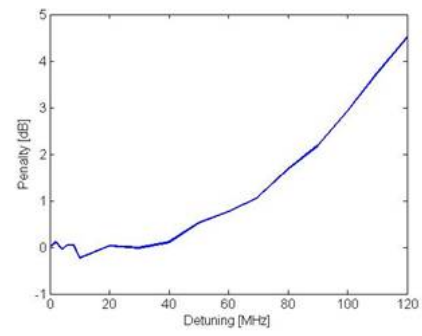

(b)

Fig. 7. (a) Receiver sensitivity for several linewidths, (b) Sensitivity penalty against frequency offset between Tx and Rx lasers

In practice, the Rx LO would be different than the Tx laser, hence frequency offset between the two light-sources would limit the performance of the Rx. Figure 7(b) plots the sensitivity penalty for a FEC target BER of $10^{-3}$ against the frequency offset obtained by simulations. It can be noticed that a $1 \mathrm{~dB}$ penalty is achieved already at $70 \mathrm{MHz}$, very close to the one observed in [9]. This indicates that the receiver is very sensitive to wavelength fluctuations.

\section{Conclusion}

We have proposed and demonstrated a PSK homodyne architecture based on a simpler phasediversity set-up using digital processing. High linewidth tolerance was experimentally achieved, enabling the use of DFB with a combined Tx/Rx linewidth of $30 \mathrm{MHz}$, representing a tolerance of $2.4 \%$ of the bit rate in an udWDM-PON scenario. Besides, the use of DSP enhances the sensitivity in about $4 \mathrm{~dB}$ compared with an analog implementation.

This new architecture is implementable using commercially available semiconductor lasers (i.e. DFB lasers) and low-cost standard components, either optical or electrical. Thus, it constitutes an enabling technique towards ultra-dense-WDM PONs, featuring few $\mathrm{GHz}$ spacing wavelengths with electrical channel filtering, simple tuning and improved sensitivity.

\section{Acknowledgments}

This work was supported in part by the European FP7 COCONUT, Conacyt grant 185291, and project TEC2011-25215 (ROMULA) of the Spanish Ministry for Science and Innovation 